\title{
BMJ Open Association between socio-economic status and dental caries in elderly people in Sichuan Province, China: a cross-sectional study
}

\author{
Linyan Wang, ${ }^{1}$ Li Cheng, ${ }^{1}$ Bo Yuan, ${ }^{1}$ Xiao Hong, ${ }^{2}$ Tao Hu ${ }^{1}$
}

To cite: Wang $L$, Cheng $L$, Yuan $\mathrm{B}$, et al. Association between socio-economic status and dental caries in elderly people in Sichuan Province, China: a crosssectional study. BMJ Open 2017;7:e016557. doi:10.1136/ bmjopen-2017-016557

- Prepublication history for this paper is available online. To view these files please visit the journal online (http://dx.doi. org/10.1136/bmjopen-2017016557)

LW and LC contributed equally.

Received 24 February 2017

Revised 14 June 2017

Accepted 22 August 2017
CrossMark

${ }^{1}$ Department of Preventive Dentistry, West China Hospital of Stomatology, Sichuan University, Chengdu, Sichuan, China

${ }^{2}$ Department of General

Dentistry, West China Hospital of Stomatology, Sichuan University, Chengdu, Sichuan, China

Correspondence to

Professor Tao Hu;

hutao@scu.edu.cn

\section{ABSTRACT}

Objectives As a vulnerable group, the elders' oral health gained less attention, particularly the relationship between socioeconomic status (SES) and dental caries. This study aimed to assess the associations and to explore the effects of confounders on the associations in elderly people.

Design Cross-sectional study.

Settings 3 neighbourhood committees and 3 village committees in Sichuan Province, China.

Participants 744 people (362 men and 382 women) aged 65-74 years were included.

Outcome measures Oral health outcomes included the decayed, missing and filled teeth (DMFT) index and its components. SES was assigned by educational level, household income and type of household. The bivariate association between the participants' characteristics and DMFT was analysed using non-parametric tests. Four logistic regression models were used to analyse the associations between SES and dental caries by regulating confounders.

Results Poor oral health was observed in these participants. Bivariate analysis showed a significant association between SES and DMFT $(p<0.05)$. Only adjusting gender, high educational level (adjusted $(\mathrm{AOR})=0.34,95 \% \mathrm{Cl} 0.17$ to 0.66 ), high household income ( $\mathrm{AOR}=0.47,95 \% \mathrm{Cl} 0.41$ to 0.77 ) were protective factors against dental caries, and living in agricultural families $(\mathrm{AOR}=1.86,95 \% \mathrm{Cl} 1.32$ to 2.63$)$ was risk factor $(\mathrm{p}<0.05)$. After adjusting other confounders, SES was partly related to the dental caries. Moreover, an interaction existed among SES indicators.

Conclusions SES is associated with dental caries, and older people with low SES have poor oral health. The associations were explained partly by diet, behaviour and awareness. Our results provide effective evidence in targeted policy-making and intervention measures and implicate that pertinence measures, economic assistance and medical insurance funds should be provided to older people of low SES. Furthermore, a follow-up design should attempt to confirm the causal relationship between SES and dental caries and evaluate the effect of intervention.

\section{INTRODUCTION}

Oral diseases, especially dental caries, periodontal disease and tooth loss, affect general health and can impair quality of life. ${ }^{1}$ The
Strengths and limitations of this study

This study focuses on the older adults in China and includes the large sample size.

- This study provides greater understanding of the relations between socioeconomic status and dental caries by multivariate logistic regression models.

For elderly people aged 65-74 years, the reason of missing teeth could not be traced.

- As a cross-sectional study, it is difficult to sort out causal relationship between socioeconomic status and dental caries.

high morbidity of dental caries increases healthcare costs and the financial burden to families and societies, which are of concern. ${ }^{2}$ Although the overall prevalence of caries and the number of decayed, missing and filled teeth (DMFT) have decreased in adolescents and adults in past decades, ${ }^{34}$ the burden associated with caries remains high in disadvantaged, poor and older populations. Schwendicke et al reviewed that those with lower educational level or occupational background, or lower income were more likely to have higher risk of caries lesions or experience. ${ }^{5}$

There is a complex relationship between personal socioeconomic status (SES) and oral health ${ }^{5-7}$ Hobdell $e t$ al showed that there is a discernible association between oral diseases and SES, and the skewed distribution of caries lesions is thought to be a good proxy measure for socioeconomic development. ${ }^{89}$ Previous studies have shown that people with a low SES have poorer oral health status than do those with a higher SES and that oral health worsens progressively from higher SES to lower SES. ${ }^{10}{ }^{11}$ SES includes educational background, income and residential area and is considered to be one of the strongest determinants of caries in children. ${ }^{12}$ Household income and educational level 
are significantly associated with periodontitis and edentate status in elderly people. ${ }^{711}$ Therefore, the literatures suggest that socioeconomic factors are crucial oral health determinants and that inequality in SES is an important challenge for public oral health.

In the Third National Survey of Oral Health Status in China in 2005, $86.0 \%$ of older people aged $65-74$ years were shown to have some degree of dental caries $^{13}$; this prevalence was higher than the figure of $64.8 \%$ noted in the Second National Survey of Oral Health Status in $1995 .^{14}$ The Third National Survey showed poor oral health status in older people. For instance, the mean number of missing teeth was 11.03 , the mean number of filled teeth was only 0.25 and $6.8 \%$ of older people exhibited full edentulism. ${ }^{13}$ In addition to the high incidence rates of the presence of residual roots and edentulism, the resulting psychological and financial burdens indicate the need for prevention and treatment in certain populations such as the elderly and those with low SES..$^{15-17}$

As we have known, demographic variables, diet, use of fluorides, oral hygiene and access to health service are related to dental caries. ${ }^{3}{ }^{18}$ Some studies have reported the effects of socioeconomic factors on oral health-related behaviours, such as encouraging health-enhancing behaviours in adolescents and dental care use in adults. ${ }^{192}$ However, there are limited nationally representative data from China on the variability in oral health status, especially in relation to SES and in the elderly population, due to issues such as small sample size and lack of rigorous design or statistical power. ${ }^{21}$ As the elderly population with variable SES, it is unclear whether the disparity in the incidence of dental caries is modified significantly by gender, dietary habits, oral health-related behaviours or health awareness. Exploring the relationships between dental caries, SES and possible contributing factors may be beneficial for developing targeted interventions and healthcare policies. ${ }^{22}$

To this end, we report here a cross-sectional analysis of data from the Survey of Oral Health Status in an elderly population in Sichuan Province, China. The objective of this study was to examine the relationships between dental caries and SES factors (type of household, income and educational level) in elderly people and to ascertain whether the associations are influenced by gender, personal habits or health awareness. Based on situational analysis of the representative data, we aim to interpret and address the inequalities observed by improving the cooperation among multisectoral stakeholders and relevant agencies.

\section{METHODS}

\section{Study design and sample}

The analysis included data from participants aged 65-74 years in Sichuan Province. With a population of 81.07 million in 2013, Sichuan Province is located in southwest China and covers an area of $485000 \mathrm{~km} .{ }^{23}$ The province suffers from inequalities in the level of economic development and distribution of healthcare resources. ${ }^{24}$ The cross-sectional national survey was conducted from December 2015 to May 2016 by the Department of Preventive Dentistry, West China Hospital of Stomatology, Sichuan University. A fourstage stratified random-cluster survey sampling method was adopted to obtain a representative sample of the residents in Sichuan Province based on the number of permanent residents and non-agricultural population ratio obtained in the 2010 Sixth National Population Census. In the first stage, two districts and two counties were chosen randomly by the stratified sampling using the probability proportional to size (PPS) method in the Fourth National Oral Health Survey in China. However, the four previously selected areas were found to concentrate mainly on the middle and low levels of urbanisation. Therefore, in the second stage, to gain a sufficiently representative sample, two extra areas were chosen from areas with high levels of urbanisation by repeating the sampling process described above. ${ }^{25}$ In the third stage, three neighbourhood committees and three village committees in each of the six areas were selected by PPS random sampling. In the fourth stage, the native specific participants were identified by quota sampling. Persons with serious physical or psychological illness or disadvantages, who were unable or unwilling complete the examination and questionnaire, were excluded. Ultimately, 744 voluntary person aged 65-74 years were surveyed; this number was greater than the expected 696 participants calculated by the formula:

$$
n=\operatorname{deff} \frac{\mu^{2}(1-p)}{\epsilon^{2} p}
$$

in which the design efficiency deff=2.5, the confidence of investigation $\mu=1.96$, the acceptable error $\varepsilon=10 \%$, the prevalence of caries in people at aged 65-74 years from the Third National Oral Health Survey $\mathrm{p}=86.0 \%$ and the non-response rate was $10 \%$.

\section{Data collection}

Data were collected through an oral examination and questionnaire in mobile examination centres. The oral examinations were conducted by four trained and accredited dentists with the assist of trained recorders, according to WHO criteria. ${ }^{26}$ The kappa values of the examiners ranged from 0.80 to 0.98 . Status of dental caries was examined with the a dental mirror, Community Periodontal Index (CPI) probe, under the artificial light, according to the standard proposed by the WHO ${ }^{26}$ Every participant was asked to fill an interviewer-administered questionnaire. Considering the illiterate participants, trained dental students conducted the interviews of questionnaires. The structured questionnaire contained sociodemographic characteristics, health-related behaviours and key sociodemographic variables. 


\section{Variables}

Oral health outcomes

The participants' dental caries status was evaluated by analysing the DMFT index and its components. Teeth were classified as decayed (DT) if there was evidence of cavitation on the crown or root. Teeth missing for whatever reason in older people were classified as missing (MT). Filled teeth without secondary caries were classified as filled (FT). ${ }^{26}$ The DMFT score was categorised according to two levels of severity: low dental caries (DMFT $\leq 19$ ) and high dental caries (DMFT $>19)$. The number of DT was categorised into two levels: low decay $(\mathrm{DT} \leq 7)$ and high decay $(\mathrm{DT}>7)$. The number of MT was classified into two groups: few missing teeth $(\mathrm{MT} \leq 10)$ and more missing teeth $(\mathrm{MT}>10)$. All variables were with the 75 th percentile of value distribution as the cut-off point. ${ }^{27}$ The number of FT was recorded and divided into two groups: with or without (yes or no).

\section{SES indicators}

A structured questionnaire was used to obtain information about SES, including household income, education and type of household. The annual household income was determined from the questionnaire item, 'What is your approximate total household income in the past 12 months?' Household income was required to be answered in the integer value of $¥ 10000$ and was categorised into quartiles: low ( $\leq ¥ 20000 /$ year, approximately US\$2903.5/year); medium ( $¥ 30000 /$ year- $¥ 40,000$ / year, approximately US\$4353.8/year-US\$5805.0/ year); medium-high ( $¥ 50000 /$ year- $¥ 70,000 /$ year, approximately US $\$ 7256.3 /$ year-US $\$ 10158.8 /$ year) and high ( $\geq ¥ 80000 /$ year, approximately US $\$ 11610.0$ / year).$^{1528}$ Because household income is a sensitive issue, the participants were allowed to leave this question unanswered. Participants who would not share their household income were excluded from analyses that involved income. As a key component of social status, the participants were asked about their highest educational attainment. This was then categorised into four levels: illiterate, low (primary school), medium (junior high school) and high (high school or above). ${ }^{29}$ The type of family household was classified as non-agricultural or agricultural.

\section{Covariates}

Several categories of covariates were included in the questionnaire.

Demographic variables:

\section{- Gender}

Consumption of desserts and sugared beverages ${ }^{411}$ :

- Frequency of desserts, including cake, bread, biscuits and sweets, classified into two levels: low (less than once a day) and high (more than once a day);

- Frequency of sugared beverages, classified into two levels: low (less than once a day) and high (more than once a day).

Oral health-promoting behaviours:
- Tooth brushing frequency, classified into twice a day or more and less than twice a day ${ }^{30}$;

- Dental flossing, determined from the question 'Do you use dental floss to clean your teeth?' and classified as yes or no ${ }^{10}$;

- Dental appointments, determined from the question 'Have you ever visited a dentist?' and classified as yes or no. ${ }^{8}$

Knowledge and attitudes:

- Twelve questions about the promotion of good oral health, the causes of oral diseases and how these play a role in the preservation of healthy teeth were included. The number of correct answers to the 12 questions was calculated for each participant, ${ }^{31}$ and the participants were classified into tertiles according to the number of correct answers as high, intermediate and low.

\section{Data analysis}

Continuous data, such as DMFT score and number of DT, MT and FT, are reported as mean \pm SD. First, a descriptive analysis was used to analyse the frequencies and percentages of dental caries and root decay. Because the outcomes were not normally distributed, non-parametric tests, including the Mann-Whitney U-test and Kruskal-Wallis $\mathrm{H}(\mathrm{K})$ test, were used to evaluate the bivariate associations between the participants' characteristics (including gender, poor health behaviours, health-promoting behaviours, health-related knowledge, dental appointments and SES) and the outcomes (DMFT score and numbers of DT, MT and FT). Multivariate logistic regression models were used to estimate the associations between the severity of DMFT and its components with educational level, household income and type of household. In the four models, various variables were added gradually to explore the effects of SES on dental caries after adjusting covariates. Initially, gender was adjusted in model 1 to confirm whether the gender affect the associations between outcomes and SES, and dietary habits were added in model 2 to explore whether the associations are influenced by consumption of desserts and sugared beverages. This was followed by additional adjustments for oral health-related behaviours and health awareness in model 3 to exclude the effects of all covariates and finally, other different SES indicators in model 4 to verify the interaction among SES indicators. The adjusted ORs (AORs) and 95\% CIs were used for regression analysis. For data analysis and statistics, SPSS Statistics V.17.0 (SPSS) was used. p Values $<0.05$ were considered to be significant.

\section{RESULTS}

\section{Sample characteristics}

A total of 744 people aged 65-74 were included: 362 (48.7\%) men and $382(51.3 \%)$ women. Participants of $741(99.6 \%)$ were ethnically Han, only 3 participants 


\begin{tabular}{|c|c|c|}
\hline Outcome $(\geq 1)$ & n (\%) & Mean \pm SD \\
\hline DMFT & 730 (98.2) & $13.32 \pm 9.58$ \\
\hline DT & $596(80.1)$ & $4.93 \pm 5.38$ \\
\hline MT & 685 (92.1) & $8.11 \pm 8.35$ \\
\hline FT & 95 (12.8) & $0.28 \pm 0.97$ \\
\hline DFRoot & $375(50.4)$ & $1.58 \pm 2.45$ \\
\hline DRoot & 367 (49.3) & $1.51 \pm 2.40$ \\
\hline FRoot & $20(2.7)$ & $0.07 \pm 0.59$ \\
\hline
\end{tabular}

DMFT, decayed, missing and filled teeth; DT, decayed teeth; MT, missing teeth; FT, filled teeth; DFRoot, decayed root; DRoot, decayed root; FRoot, filled root.

were minority. Surprisingly, only nine participants (1.2\%) flossed. Nearly all $(98.2 \%, \mathrm{n}=730)$ participants had caries $($ DMFT $\geq 1)$ and the mean DMFT score $( \pm \mathrm{SD})$ was $13.32 \pm 9.58$. It is worth reminding that $16.5 \% \quad(n=123)$ illiterate participants were included in the samples, all of them suffered from dental caries and their mean DMFT score $( \pm \mathrm{SD})$ was $16.20 \pm 10.23$. As a component of DMFT, the prevalence rate for DT $\geq 1$ was $80.1 \%(n=596)$ and the mean number of DT was $4.93 \pm 5.38$. The mean number of MT was $8.11 \pm 8.35,8.7 \%(\mathrm{n}=65)$ of participants were even edentulous-no teeth in the mouth, and the mean number of existed teeth $( \pm \mathrm{SD})$ of participants was $23.89 \pm 8.35$. The mean number of FT was only $0.28 \pm 0.97$. Half of the participants $(50.4 \%, \mathrm{n}=375)$ had at least 1 decayed and filled root, just under half had at least 1 decayed root, but only 20 people had at least 1 well-filled root (table 1 ).

\section{Bivariate analysis}

The distribution of DMFT according to different exposure variables is shown in table 2 . The inequality in the DMFT score distribution was associated with gender, brushing frequency, history of dental appointments, type of household, educational level and household income. The number of DT was related to brushing frequency, type of household, educational level and oral health-related knowledge. The number of MT was related to history of dental appointments, type of household and educational level. The number of FT was related to brushing frequency, history of dental appointments, type of household, educational level, household income and oral health-related knowledge.

\section{SES indicators}

\section{Education}

An important marker of SES, educational level, is significantly associated with dental caries in elderly people. ${ }^{32}$ As to DMFT, figure 1 shows that, in the gender-adjusted model (model 1$)$, participants with a medium $(\mathrm{AOR}=0.53$, $95 \%$ CI 0.30 to $0.95, \mathrm{p}=0.033)$ or high $(\mathrm{AOR}=0.34,95 \%$ CI 0.17 to $0.66, \mathrm{p}=0.002$ ) educational level had significantly lower AORs compared with the illiterate group (as reference, $A O R=1)$. After adjusting for oral health-related behaviours and health awareness (model 3), the AORs of DMFT were not significant in the medium educational level group ( $\mathrm{AOR}=0.55,95 \% \mathrm{CI} 0.29$ to $1.05, \mathrm{p}=0.259$ ), but the AORs in the high educational level group $(\mathrm{AOR}=0.39$, $95 \%$ CI 0.18 to $0.83, p=0.049$ ) remained significantly lower until the fully adjusted model (model $4, \mathrm{AOR}=0.67,95 \% \mathrm{CI}$ 0.29 to $1.51, \mathrm{p}=0.328$ ). As to DT and MT, the AORs were significantly lower in the group with high educational level in model 1 (DT: AOR $=0.33,95 \%$ CI 0.17 to $0.67, \mathrm{p}=0.002$; MT: AOR $=0.45,95 \%$ CI 0.23 to $0.88, \mathrm{p}=0.019$ ) and model 2 (DT: $\mathrm{AOR}=0.38,95 \%$ CI 0.17 to $0.68, \mathrm{p}=0.002$; MT: $\mathrm{AOR}=0.45,95 \%$ CI 0.23 to $0.89, \mathrm{p}=0.021$ ), then were close to one after adjusting for dental appointments, brushing frequency and health awareness (model 3, DT: AOR $=0.48$, 95\% CI 0.20 to $0.94, \mathrm{p}=0.022$; $\mathrm{MT}$ : $\mathrm{AOR}=0.51,95 \% \mathrm{CI}$ 0.24 to $1.08, \mathrm{p}=0.051$ ). Educational level was associated with the number of FT after adjusting for gender, dietary habits, oral health-related behaviours and health awareness (models $1-3, \mathrm{p}<0.05$ ). This association was explained by household income and type of household in the group with lower educational level (low: AOR $=1.94,95 \%$ CI 0.63 to $6.01, \mathrm{p}=0.235$; medium: $\mathrm{AOR}=2.57,95 \%$ CI 0.76 to 8.60 , $\mathrm{p}=0.119$ ), whereas high educational level was still associated with the number of FT even in the fully adjusted model (model $4, \mathrm{AOR}=4.43,95 \%$ CI 1.27 to $15.46, \mathrm{p}=0.018$ ).

\section{Household income}

The mean DMFT differed as the annual household income varied. Compared with the low household income, multiple logistic regression analysis showed a significant association between DMFT and medium-high and high-income in model 1 (medium-high: AOR $=0.51$, 95\% CI 0.34 to $0.83, \mathrm{p}=0.007$; high: $\mathrm{AOR}=0.47,95 \%$ CI 0.41 to $0.77, \mathrm{p}=0.043$ ) and model 2 (medium-high: $\mathrm{AOR}=0.51,95 \% \mathrm{CI} 0.31$ to $0.84, \mathrm{p}=0.007$; high: $\mathrm{AOR}=0.46$, $95 \%$ CI 0.39 to $0.78, \mathrm{p}=0.043$ ). These associations could be explained by oral health-enhancing behaviours, better knowledge and health awareness (model 3, mediumhigh: $\mathrm{AOR}=0.68,95 \%$ CI 0.41 to $1.14, \mathrm{p}=0.062$; high: $\mathrm{AOR}=0.90,95 \%$ CI 0.53 to $1.15, \mathrm{p}=0.154$ ) (figure 2). Household income was not significantly associated with any DMFT component in the four models, except for the FT. As to FT, the AORs respectively were 3.12 (95\% CI 1.42 to $6.82, \mathrm{p}=0.004$ ) in medium group, 4.80 (95\% CI 2.26 to $10.18, \mathrm{p}<0.000)$ in medium-high group and $5.86(95 \%$ CI 2.76 to $12.43, \mathrm{p}<0.001)$ in high group. And in model 2, the AORs were 3.17 (95\% CI 1.45 to $6.94, \mathrm{p}=0.004$ ) in medium group, 4.93 (95\% CI 2.92 to $10.49, \mathrm{p}<0.001)$ in medium-high group and 5.72 (95\% CI 2.71 to 12.09 , $\mathrm{p}<0.001)$ in high group. The AORs of different groups decreased gradually to one after adjusting for different covariates and were significant in model 3 (medium: $\mathrm{AOR}=2.28,95 \%$ CI 1.02 to $5.12, \mathrm{p}=0.026$; medium-high: $\mathrm{AOR}=3.38,95 \%$ CI 1.54 to $7.42, \mathrm{p}<0.001$; high: $\mathrm{AOR}=3.16$, $95 \%$ CI 1.43 to $6.97, \mathrm{p}=0.002)$. In the fully adjusted model (model 4), household income was not significantly associated with FT after adjusting for educational level and type of household ( $p>0.05)$. 
Table 2 Descriptive information and bivariate associations

\begin{tabular}{|c|c|c|c|c|c|c|c|}
\hline \multirow[b]{2}{*}{ Variable } & \multirow[b]{2}{*}{$\mathbf{n}$} & \multirow{2}{*}{$\begin{array}{l}\text { DMFT>0\% } \\
\text { n (\%) }\end{array}$} & \multirow{2}{*}{$\begin{array}{l}\text { DMFT } \\
\text { (mean } \pm S D)\end{array}$} & \multicolumn{4}{|l|}{ p Value } \\
\hline & & & & DMFT & DT & MT & FT \\
\hline \multicolumn{8}{|l|}{ Gender } \\
\hline Female & 382 & $378(99.0)$ & $13.95 \pm 9.68$ & & & & \\
\hline \multicolumn{8}{|l|}{ Consumption of desserts } \\
\hline$<1 /$ day & 529 & $520(98.3)$ & $13.47 \pm 9.62$ & & & & \\
\hline \multicolumn{8}{|c|}{ Consumption of sugared beverages } \\
\hline$\geq 1 /$ day & 249 & 247 (99.2) & $13.10 \pm 9.43$ & $0.459^{\star}$ & $0.626^{*}$ & $0.554^{*}$ & $0.329^{*}$ \\
\hline$<1 /$ day & 495 & $483(97.6)$ & $13.75 \pm 9.88$ & & & & \\
\hline \multicolumn{8}{|l|}{ Brushing } \\
\hline Never & 71 & $71(100.0)$ & $19.79 \pm 10.96$ & & & & \\
\hline \multicolumn{8}{|l|}{ Dental appointments } \\
\hline Yes & 553 & 547 (98.9) & $13.18 \pm 9.68$ & $<0.001^{*}$ & $0.061^{*}$ & $<0.001^{*}$ & $<0.001^{*}$ \\
\hline No & 191 & $183(95.8)$ & $10.77 \pm 8.73$ & & & & \\
\hline \multicolumn{8}{|l|}{ Health awareness } \\
\hline Low & 236 & $229(97.0)$ & $14.20 \pm 9.92$ & $0.192 \dagger$ & $0.020 \dagger$ & $0.205 \dagger$ & $<0.001 \dagger$ \\
\hline Medium & 273 & 268 (98.2) & $13.6 \pm 9.68$ & & & & \\
\hline High & 275 & 273 (99.3) & $12.51 \pm 9.07$ & & & & \\
\hline Low & 228 & $228(100.0)$ & $13.53 \pm 9.60$ & $0.015 \dagger$ & $0.051 \dagger$ & $0.173 \dagger$ & $<0.001 \dagger$ \\
\hline Medium & 165 & $160(97.0)$ & $13.35 \pm 9.52$ & & & & \\
\hline Medium-high & 163 & $159(97.6)$ & $13.18 \pm 9.41$ & & & & \\
\hline High & 149 & $146(98.0)$ & $12.48 \pm 9.71$ & & & & \\
\hline \multicolumn{8}{|l|}{ Type of household } \\
\hline Non-agricultural family & 340 & $332(97.7)$ & $11.80 \pm 8.65$ & $<0.001^{*}$ & $0.002^{*}$ & $0.007^{\star}$ & $<0.001^{*}$ \\
\hline Agricultural family & 404 & $398(98.5)$ & $14.60 \pm 10.14$ & & & & \\
\hline
\end{tabular}

*Mann-Whitney U-test.

†Kruskal-Wallis $\mathrm{H}(\mathrm{K})$ test.

$\ddagger$ Subjects did not report household income were excluded, included participants $n=705$.

DMFT, decayed, missing and filled teeth; DT, decayed teeth; FT, filled teeth; MT, missing teeth.

Type of household

The AORs of DMFT were higher in participants from agricultural families than those from non- agricultural families in the models after adjusting for gender (model 1, $\mathrm{AOR}=1.86,95 \%$ CI 1.32 to $2.63, \mathrm{p}<0.001)$, dietary habits (model $2, \mathrm{AOR}=1.88,95 \%$ CI 1.33 to $2.67, \mathrm{p}<0.001$ ), oral health-related behaviours and health awareness (model 3, $\mathrm{AOR}=1.61,95 \% \mathrm{CI} 1.08$ to $2.42, \mathrm{p}<0.001$ ), and these associations were explained after adjusting for household income and educational level (model 4, AOR=1.52, $95 \%$ CI 0.98 to $2.37, \mathrm{p}=0.054$ ), as shown in figure 3 . As to DT and MT, the AORs were significantly higher in participants from agricultural families in model 1 (DT: $\mathrm{AOR}=1.67,95 \%$ CI 1.17 to $2.34, \mathrm{p}=0.004$; $\mathrm{MT}$ : $\mathrm{AOR}=1.55$, $95 \% \mathrm{CI} 1.10$ to $2.19, \mathrm{p}=0.012$ ) and model 2 (DT: $\mathrm{AOR}=1.64,95 \%$ CI 1.16 to 2.33, $\mathrm{p}=0.005$; MT: $\mathrm{AOR}=1.55$, 


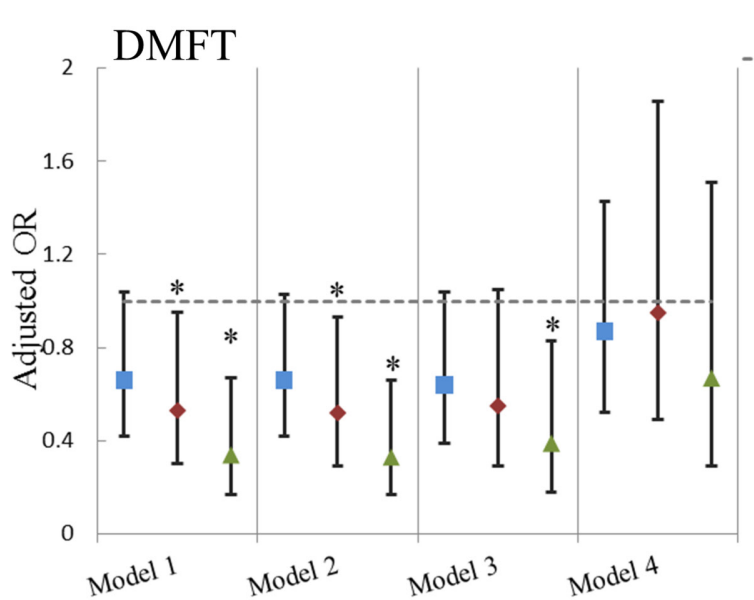

MT
DT

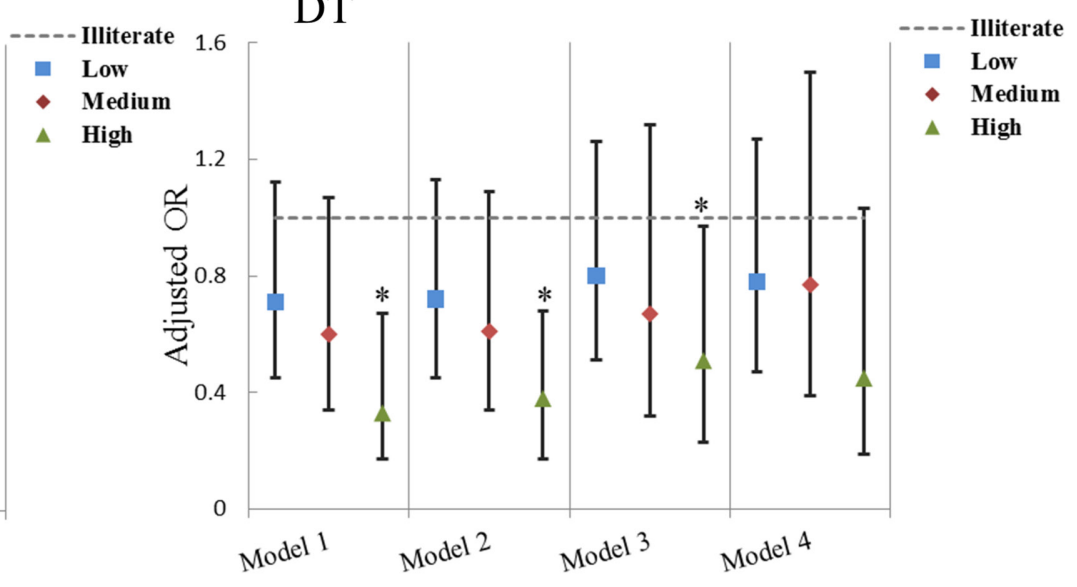

FT

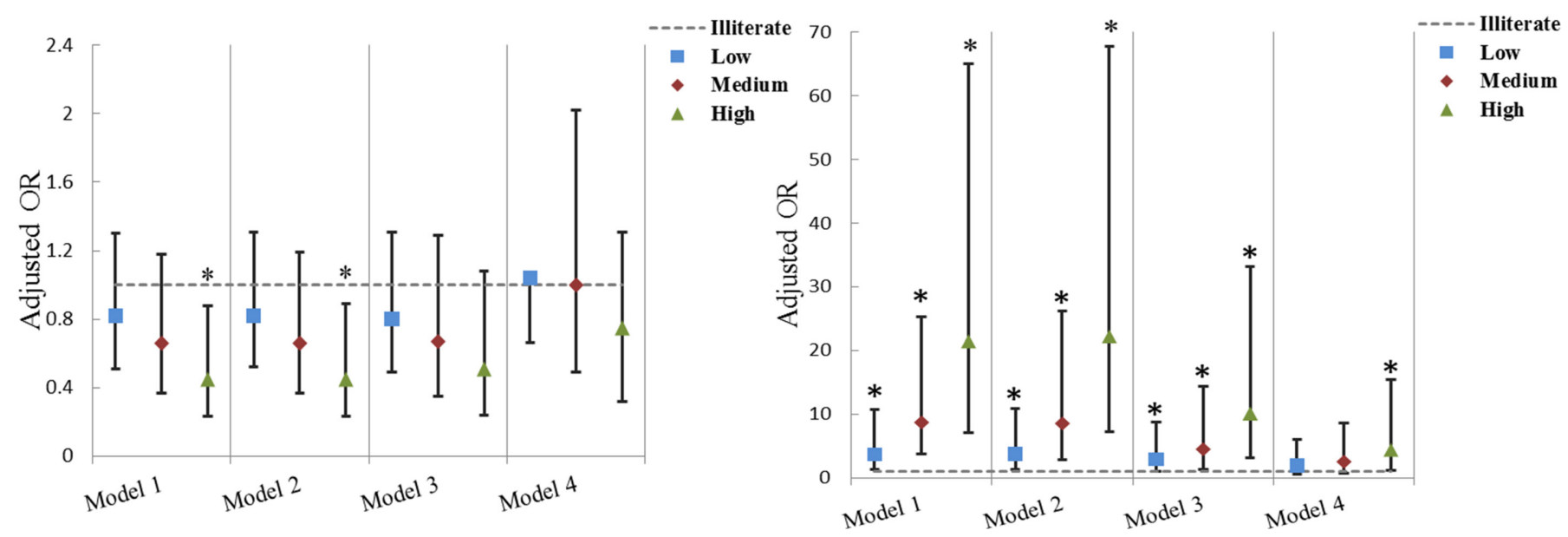

Figure 1 Associations between educational level and the severity of DMFT (low dental caries, high dental caries), DT (low decay, high decay), MT (few missing teeth, more missing teeth) and FT (with, without). The data were analysed using logistic regression models and are presented as adjusted ORs referenced to illiterate. The bars indicate the $95 \% \mathrm{Cl}$. ${ }^{*} \mathrm{p}<0.05$. Model 1 was adjusted for education and gender. Model 2 was adjusted for education, gender and consumption of desserts and sugared beverages. Model 3 was adjusted for education, gender, consumption of desserts and sugared beverages, dental appointments, brushing frequency and oral health-related awareness. Model 4 was adjusted for education, gender, consumption of desserts and sugared beverages, dental appointments, brushing frequency, oral health-related awareness, household income and type of household. DMFT, decayed, missing and filled teeth; DT, decayed; MT, missong teeth; FT, filled teeth.

95\% CI 1.10 to $2.19, \mathrm{p}=0.013$ ), and these associations were explained by oral health-related behaviours and health awareness. However, as shown in figure 3, for FT, the AORs were significantly lower in participants from agricultural families in all adjusted models (model 1 : AOR $=0.10$, $95 \%$ CI 0.06 to 0.19 ; model 2: AOR $=0.10,95 \%$ CI 0.06 to 0.19; model 3: AOR $=0.17$, $95 \%$ CI 0.09 to 0.33 ; model 4 : $\mathrm{AOR}=0.18,95 \%$ CI 0.08 to 0.37 ; all the $\mathrm{p}<0.001$ ).

\section{DISCUSSION}

This study provides systematic information about dental caries in people aged 65-74 years in Sichuan Province. The oral health status of children and adolescents has been widely studied. However, there are few local and national studies on adults, especially elderly people. ${ }^{33-35}$ The high prevalence rates of caries and history of caries, as shown by the number of DMFT, DT and MT in 2016 were similar to those reported in Uruguay, ${ }^{36}$ Chile $^{37}$ and in the 2005 survey in China. ${ }^{1238}$ The high rates of MT and root decay show that older people in Sichuan Province have poor oral health. The low number of FT indicates a high rate of untreated DT. Consistent with the poor oral health condition in these participants, this epidemiological survey found a lack of general knowledge, awareness and behaviour related to oral healthcare. For example, only $32.8 \%$ of the $65-74$-year-old participants in this study brushed their teeth at least twice a day and only $1.2 \%$ flossed. The worse oral hygiene habits reminded us of the importance of the healthy and effective oral healthcare behaviours, such as proper tooth brushing, universal flossing and regular oral health examination. In a word, there is still a long way to go to improve oral health in older people in this region of China. 


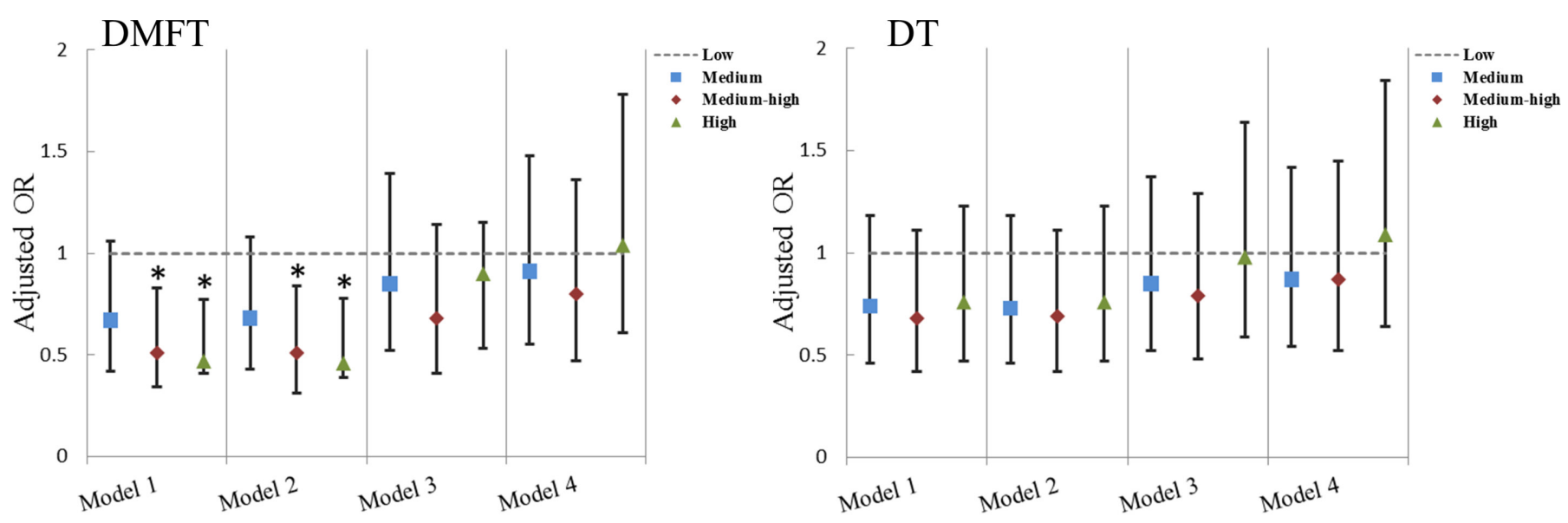

damaged teeth, unreplaced extracted teeth and periodontal diseases. ${ }^{103940}$ By adjusting covariates gradually, the multivariate logistic regression models indicated that three indicators of SES-educational level, household income and type of household-were related to the experience of dental caries in different ways.

Educational level, as a traditional SES variable, affects the type of job and income, and thus access to preventive measures such as tooth cleaning habits, health service use and a low-carbohydrate diet. ${ }^{41} 42$ In our study, an educational level higher than primary school was a protective factor against dental caries, and the higher the educational level, the stronger was the protective effect. Those with a high school background or above were less likely to have DT and MT. The attenuated association in model 3 indicated that the protective effect be a determinant in caries risk assessment. People with low SES are more likely to present with dental pain, 

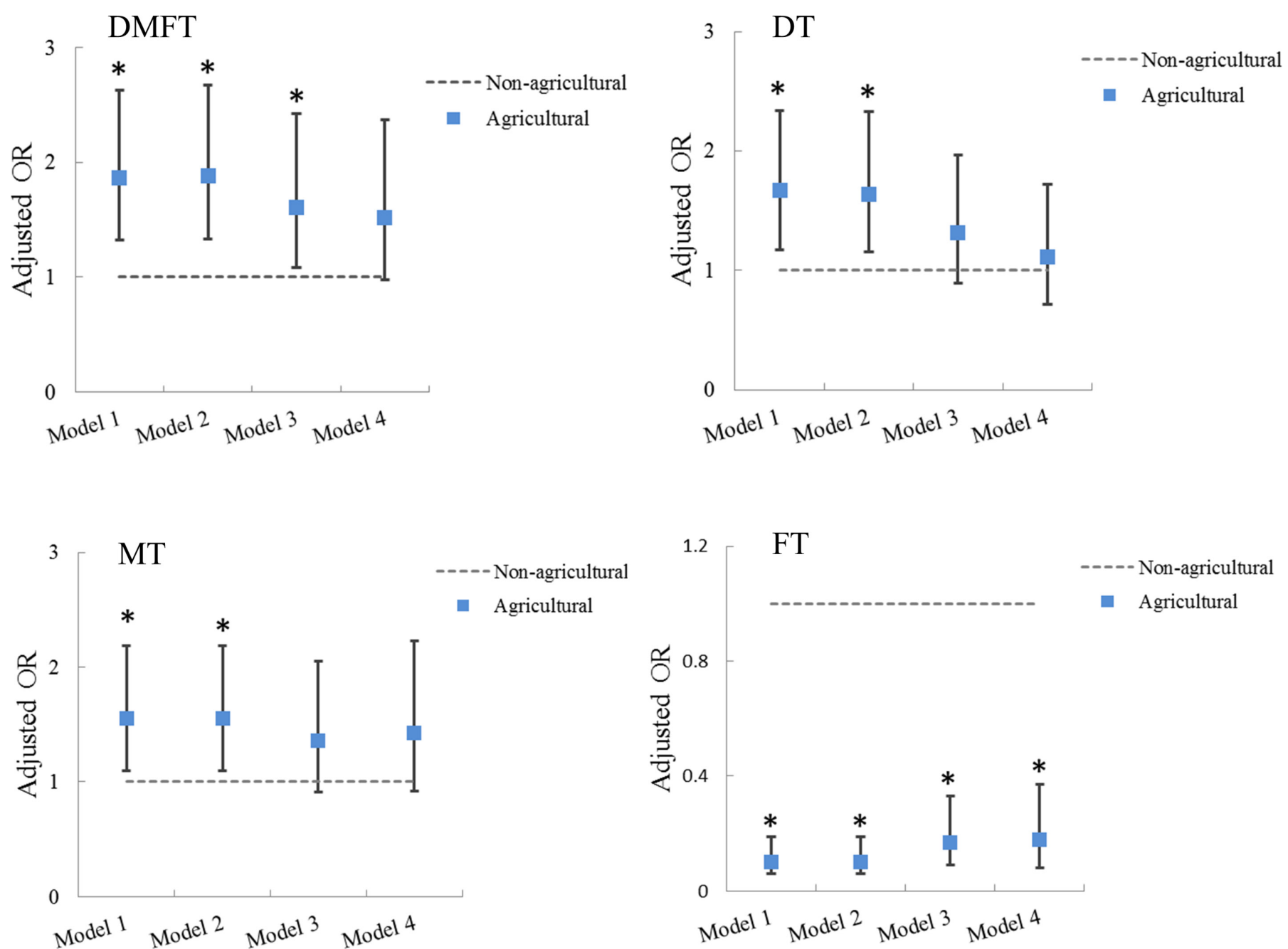

Figure 3 Associations between type of household and the severity of DMFT (low dental caries, high dental caries), DT (low decay, high decay), MT (few missing teeth, more missing teeth) and FT (with, without). The data were analysed using logistic regression models and are presented as adjusted ORs referenced to non-agricultural families. The bars indicate the $95 \% \mathrm{Cl}$. DT, MT and FT were significantly associated with the type of household. ${ }^{*} p<0.05$. Model 1 was adjusted for type of household and gender. Model 2 was adjusted for type of household, gender and consumption of desserts and sugared beverages.

Model 3 was adjusted for type of household, gender, consumption of desserts and sugared beverages, dental appointments, brushing frequency and oral health-related awareness. Model 4 was adjusted for type of household, gender, consumption of desserts and sugared beverages, dental appointments, brushing frequency, oral health-related awareness, educational level and household income. DMFT, decayed, missing and filled teeth; DT, decayed teeth; MT, missing teeth; FT, filled teeth.

was partly explained by oral health knowledge and behaviours. The significant AORs of FT indicated that participants with any educational level higher than illiterate were more likely to seek professional help and to make better use of medical services and information. After controlling for the type of household and income, educational level was unrelated to the severity of DMFT, which suggests that education may be the dominant factor of SES. ${ }^{41}$ Ghorbani and Peres found that people with a lower educational level were more likely to have tooth extractions without receiving a prosthesis. ${ }^{40}$ Steele et al also reported that lower educational attainment was closely related to the poorest clinical outcomes, including the presence of decay, existence of unrestored decay and deep periodontal pockets. ${ }^{9}$ These findings indicate the need for programmes targeted at people with low educational level to encourage them to receive appropriate treatment.

In the logistic regression analysis, family income affected mainly the number of FT: the higher the family income, the higher was the number of FT. This relationship was partly mediated by oral health-related behaviours and health awareness. Generally, income has a direct effect on material resources and may subsequently affect clinical decisions and the ability to pay for services. ${ }^{43}$ After adjusting for educational level and type of household, the effects of income disappeared. This result shows that the effects of income inequality on dental caries appear to be mediated largely by educational and demographic factors. 
The significant effects of type of household on healthcare outcomes were described above. Participants from agricultural families were more likely to have poor oral health because of their poor health-related behaviours and health awareness. The DMFT score and number of MT were explained by the inequalities in educational level and household income. The association between the number of FT and type of household remained in the fully adjusted model, which may reflect the scarcity of healthcare resources for agricultural families. Other studies have shown that children and adolescents from non-agricultural families have regular oral hygiene habits compared with agricultural families. ${ }^{24}{ }^{30}$ The spatial accessibility in relation to the population distribution and transportation infrastructure affects access to healthcare. ${ }^{22} 44$

Therefore, the correlations among dental caries, SES indicators and various covariates were verified in the study by using different adjusted models. Understanding the disparities of SES and active covariates affected by different SES indicators will help organisations to adjust health education programmes to adapt to the local conditions and to target disadvantaged regions and priority populations. ${ }^{45}$

\section{Limitations}

The study had several limitations. First, it was unable to identify the reasons of missing teeth or judge whether the missing caused by caries or periodontal diseases in elderly people. Second, dental caries are caused by multiple factors and SES is manifested as various aspects. However, the study only discussed some of these factors. Finally, the reporting bias was existed in questionnaires because of the different comprehension of participants. Therefore, our results should be interpreted considering these limitations.

\section{CONCLUSION}

SES variables play a crucial role in explaining dental caries outcomes. High educational level, high household income and living in a non-agricultural family were protective against dental caries. Educational level and type of household were significantly associated with the severity of DMFT and its components through oral health-related behaviours and health awareness, and household income mainly affected the number of FT. Educational level, household income and type of household interacted with each other in influencing dental caries. Prevention is the most cost-effective approach for reducing the prevalence of dental caries. ${ }^{46}$ The preventive strategies should focus on educating people about the consequences of oral diseases and on strengthening oral health education, particularly target at the population with low educational level, low household income and from agricultural families. Our study provided evidences that the government should provide economic assistance and medical insurance funds to cover oral diseases for populations with patients of low SES. Furthermore, the research with a follow-up design is needed to confirm the causal relationship between SES and dental caries and evaluate the effect of targeted intervention.

Acknowledgements We acknowledge the technical assistance of the Sichuan Center for Disease Control and Prevention, and the statistical analysis provided by Xing Zhao and Yunyun Wu.

Contributors All authors contributed to the study design. BY collected and organised data. LW and LC undertook data analysis and drafted the manuscript. TH and $\mathrm{XH}$ undertook critical revision of the manuscript. All authors read and approved this manuscript version for submission.

Funding This study was funded by a grant from Scientific Research in the Public Interest (No. 201502002) and the National Natural Science Foundation of China (No. 81670980) for conducting the epidemical survey.

Competing interests None declared.

Patient consent Otained.

Ethics approval Ethical approvals were obtained from the Stomatological Ethics Committee of the Chinese Stomatological Association and the Ethics Committee of West China Hospital of Stomatology, Sichuan University (Approval No. 2014-003), and all participants were required to sign an informed consent form.

Provenance and peer review Not commissioned; externally peer reviewed.

Data sharing statement No additional data are available.

Open Access This is an Open Access article distributed in accordance with the Creative Commons Attribution Non Commercial (CC BY-NC 4.0) license, which permits others to distribute, remix, adapt, build upon this work non-commercially, and license their derivative works on different terms, provided the original work is properly cited and the use is non-commercial. See: http://creativecommons.org/ licenses/by-nc/4.0/

(C) Article author(s) (or their employer(s) unless otherwise stated in the text of the article) 2017. All rights reserved. No commercial use is permitted unless otherwise expressly granted.

\section{REFERENCES}

1. White DA, Tsakos G, Pitts NB, et al. Adult Dental Health Survey 2009: common oral health conditions and their impact on the population. Br Dent J 2012;213:567-72.

2. Marcenes W, Kassebaum NJ, Bernabé E, et al. Global burden of oral conditions in 1990-2010: a systematic analysis. J Dent Res 2013;92:592-7.

3. Pitts N, Amaechi B, Niederman R, et al. Global oral health inequalities: dental caries task group-research agenda. Adv Dent Res 2011;23:211-20.

4. Do LG, Lg D. Distribution of caries in children: variations between and within populations. J Dent Res 2012;91:536-43.

5. Schwendicke F, Dörfer CE, Schlattmann P, et al. Socioeconomic inequality and caries: a systematic review and meta-analysis. J Dent Res 2015;94:10-18.

6. Listl S. Income-related inequalities in dental service utilization by Europeans aged 50+. J Dent Res 2011;90:717-23.

7. Han DH, Khang YH, Choi HJ. Association of parental education with tooth loss among Korean Elders. Community Dent Oral Epidemiol 2015;43:489-99.

8. Hobdell MH, Oliveira ER, Bautista R, et al. Oral diseases and socioeconomic status (SES). Br Dent J 2003;194:91-6. discussion 88.

9. Lalloo R, Myburgh NG, Hobdell MH. Dental caries, socioeconomic development and national oral health policies. Int Dent $J$ 1999;49:196-202.

10. Steele J, Shen J, Tsakos G, et al. The Interplay between socioeconomic inequalities and clinical oral health. J Dent Res 2015;94:19-26.

11. Kim DW, Park JC, Rim TT, et al. Socioeconomic disparities of periodontitis in Koreans based on the KNHANES IV. Oral Dis 2014;20:551-9.

12. Chi DL, Masterson EE, Carle AC, et al. Socioeconomic status, food security, and dental caries in US children: mediation analyses of data from the National Health and Nutrition Examination Survey, 20072008. Am J Public Health 2014;104:860-4.

13. Xq $\mathrm{Q}$. The third national oral health epidemiological survey report. Beijing: People's Medical Publishing House 2008:34 92and. 
14. Wang HY, Petersen PE, Bian JY, et al. The second national survey of oral health status of children and adults in China. Int Dent $J$ 2002;52:283-90.

15. Wu B, Hybels C, Liang J, et al. Social stratification and tooth loss among middle-aged and older Americans from 1988 to 2004 Community Dent Oral Epidemiol 2014;42:495-502.

16. Tsakos G, Sabbah W, Chandola T, et al. Social relationships and oral health among adults aged 60 years or older. Psychosom Med 2013;75:178-86.

17. Tsakos G, Demakakos P, Breeze E, et al. Social gradients in oral health in older adults: findings from the English longitudinal survey of aging. Am J Public Health 2011;101:1892-9.

18. Cornejo-Ovalle M, Paraje G, Vásquez-Lavín F, et al. Changes in socioeconomic inequalities in the use of dental care following major healthcare reform in Chile, 2004-2009. Int J Environ Res Public Health 2014;42:536-42

19. Cho HJ, Lee HS, Paik DI, et al. Association of dental caries with socioeconomic status in relation to different water fluoridation levels. Community Dent Oral Epidemiol 2014;42:536-42.

20. Zhang Q, Li Z, Wang C, et al. Prevalence and predictors for periodontitis among adults in China, 2010. Glob Health Action 2014;7:24503.

21. Seerig LM, Nascimento GG, Peres MA, et al. Tooth loss in adults and income: systematic review and meta-analysis. J Dent 2015;43:1051-9.

22. Fisher-Owens SA, Soobader MJ, Gansky SA, et al. Geography matters: state-level variation in children's oral health care access and oral health status. Public Health 2016;134:54-63.

23. Ma Y, Zhang T, Liu L, et al. Spatio-Temporal Pattern and SocioEconomic Factors of Bacillary Dysentery at County Level in Sichuan Province, China. Sci Rep 2015:5:15264.

24. Pan J, Liu H, Wang X, et al. Assessing the spatial accessibility of hospital care in Sichuan Province, China. Geospat Health 2015;10:384.

25. Yin W, Yang YM, Chen H, et al. Oral health status in Sichuan Province: findings from the oral health survey of Sichuan, 2015-2016. Int J Oral Sci 2017;9:10-15.

26 Oral health surveys: basic methods. 5th edition. France: World Health Organization, 2013:44-6.

27 Polk DE, Weyant RJ, Manz MC. Socioeconomic factors in adolescents' oral health: are they mediated by oral hygiene behaviors or preventive interventions? Community Dent Oral Epidemiol 2010;38:1-9.

28 Tsakos G, Sabbah W, Chandola T, et al. Social relationships and oral health among adults aged 60 years or older. Psychosom Med 2013;75:178-86.

29 Dohrenwend BP, Levav I, Shrout PE, et al. Socioeconomic status and psychiatric disorders: the causation-selection issue. Science 1992;255:946-52.

30 Crocombe LA, Stewart JF, Brennan DS, et al. Is poor access to dental care why people outside capital cities have poor oral health? Aust Dent J 2012;57:477-85.
31 Zhu L, Petersen PE, Wang HY, et al. Oral health knowledge, attitudes and behaviour of adults in China. Int Dent $J$ 2005;55:231-41.

32 Jordan RA, Bodechtel C, Hertrampf K, et al. The Fifth German Ora Health Study (Fünfte Deutsche Mundgesundheitsstudie, DMS V) rationale, design, and methods. BMC Oral Health 2014;14:161.

33 Zhang S, Lo EC, Liu J, et al. A review of the dental caries status of ethnic minority children in China. J Immigr Minor Health 2015;17:285-97

34 Zhang S, Liu J, Lo EC, Ec L, et al. Dental caries status of Bulang preschool children in Southwest China. BMC Oral Health 2014;14:16.

35 Wulaerhan J, Abudureyimu A, Bao XL, et al. Risk determinants associated with early childhood caries in Uygur children: a preschool-based cross-sectional study. BMC Oral Health 2014:14:136.

36 Álvarez L, Liberman J, Abreu S, et al. Dental caries in Uruguayan adults and elders: findings from the first Uruguayan National Oral Health Survey. Cad Saude Publica 2015;31:1663-72.

37 Urzua I, Mendoza C, Arteaga O, et al. Dental caries prevalence and tooth loss in chilean adult population: first national dental examination survey. Int J Dent 2012;2012:1-6.

38 Cao CF. [Prevalences of periodontal diseases and dental caries in China: re-analysis of the data from the Third National Epidemiological Survey on Oral Health]. Zhonghua Kou Qiang Yi Xue Za Zhi 2013;48:257-9.

39 Dye BA, Tan S, Smith V, et al. Trends in oral health status: United States, 1988-1994 and 1999-2004. Vital Health Stat 11 2007;11:1-92.

40 Ghorbani Z, Peres KG. Is the association between socioeconomic status and nonreplaced extracted teeth mediated by dental care behaviours in adults? Community Dent Oral Epidemiol 2015;43:532-9

41 Shi J, Zhang Y, Liu F, et al. Associations of educational attainment, occupation, social class and major depressive disorder among Han Chinese women. PLoS One 2014;9:e86674.

42 Drewnowski A, Specter SE. Poverty and obesity: the role of energy density and energy costs. Am J Clin Nutr 2004;79:6-16.

43 Capurro DA, lafolla T, Kingman A, et al. Trends in income-related inequality in untreated caries among children in the United States: findings from NHANES I, NHANES III, and NHANES 1999-2004. Community Dent Oral Epidemiol 2015;43:500-10.

44 de Silva-Sanigorski A, Ashbolt R, Green J, et al. Parental self-efficacy and oral health-related knowledge are associated with parent and child oral health behaviors and self-reported oral health status. Community Dent Oral Epidemiol 2013;41:345-52.

45 Huang Y, Zhou K, Li H, et al. Knowledge, attitudes, and behaviour regarding breast cancer screening among women from different socio-economic regions in southwest China: a cross-sectional study. Asian Pac J Cancer Prev 2011;12:203-9.

46 Song X, Xie L, Tan X, et al. A multi-center, cross-sectional study on the burden of infectious keratitis in China. PLoS One 2014;9:e113843. 